\section{Skepticism surrounds triple Nobel winners}

For those who have not yet heard, the Nobel Assembly at the Karolinska Institute has awarded The Nobel Prize in physiology or medicine for 2000 jointly to Arvid Carlsson of the University of Goeteborg, Paul Green gard of The Rockefeller University and Eric Kandel of Columbia University. And for yet another year, the selection has stimulated controversy amongst those 'in the know' in the field of neuroscience.

The three are leading scientists in neuronal signaling, and their research into the functions of signal transduction proteins in movement control, learning and memory have led to

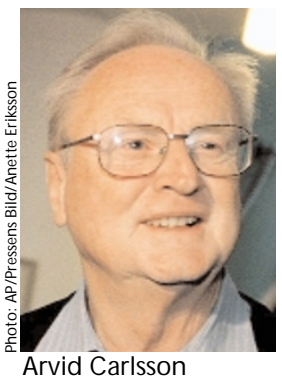

naling pathways by which neurotransmitters such as dopamine, noradrenaline and serotonin control neuronal excitability. He identified a number of signal tranduction proteins, particularly kinases and phosphatases, that are involved in synaptic transmission.

Kandel was honored for advancing the understanding of the molecular basis of learning and memory. His early work using the sea slug Aplysia demonstrated that changes in synaptic form and function are the basis of learning and memory. He new therapeutic approaches for a variety of neurodegenerative diseases and psychiatric disorders.

Carlsson won the prize for his discovery that dopamine is a neurotransmitter produced in the basal ganglia, a brain region involved in movement control. He showed that animals depleted of dopamine lose their ability to perform spontaneous movements, but that treatment with the dopamine precursor L-dopa restores normal motor behavior. Carlsson realized that the symptoms of the dopamine-depleted animals are similar to those of patients with Parkinson disease, leading to the finding that these patients have low levels of dopamine in the basal ganglia and can be treated with L-dopa.

Greengard was recognized for his contributions to the elucidation of the sig- showed that cellular signaling events such as ion channel conduction and synaptic neurotransmitter release are involved in 'short-term memory', whereas cAMP signaling and new protein synthesis are required for "long-term memory'.

One neuroscientist who talked to $\mathrm{N}$ ature Medicine said "Kandel showed that we can attack a problem as complicated as the molecular basis of learning and memory in a simple system." Kandel spent the 1990s showing that the changes in synaptic function that take place during learning in Aplysia also occur in mammals.

Although members of the neuroscience community are pleased that the importance of neuronal signaling and the involvement of these processes in learning and memory have been recognized by the Nobel committee, many also feel the combination of Carlsson, Greengard and Kandel is a peculiar one, as this year's prize covers a much broader spectrum of science than previous years' prizes.

"The Nobel prize is usually awarded for a specific breakthrough that everyone associates with one or two individuals-when you think of the patch-clamp technique you think of Erwin Neher and Bert Sakmann, when you think of the prion story, you think of Stanley Prusiner," says one neuroscientist. He explained that this is the first time that the Nobel prize has been awarded for a concept as broad as 'signal transduction in the nervous system', a concept that could be associated with dozens of different systems and hundreds of prominent scientists. "Its almost as if the committee had these three people in mind and then had to come up with a topic to encompass them all," says another researcher.

In addition, the field of learning and memory is now in a state of flux, with much individual disagree ment. Some researchers feel that the selection of Kandel-one of the most visible and influential figures in neuroscience-was premature, as many of his theories have not yet been substantiated.

Kristine Novak, New York

\section{Abortion drug approval opens up research}

With the long-awaited approval of the abortion pill mifepristone in the US, scientists are hoping that the climate of public opinion will now be more amenable to studying its other medical properties.

As far back as 1993, a panel of the National Academy of Science's Institute of Medicine called for extensive studies of the drug for other health benefits. However, the ardent antagonism generated by abortion foes meant that the drug was too tightly controlled, until now, to permit additional studies.

"[The drug] has tremendous potential," says Eric Schaff, professor of family medicine at the University of Rochester, who has studied its effect against uterine fibroid tumors, a condition affecting one-third of all women older than 40 and a leading cause of hysterectomies.

Other researchers who have examined the drug in small-scale trials have been encouraged by its potential against meningiomas of the brain and spinal cord and certain breast, ovarian and

\section{prostate cancers.}

The drug is also effective in Cushing syndrome, which results from overproduction of the hormone cortisol-mifespristone binds to glucocorticoid receptors and prevents the cortisol from binding. "I also think it has tremendous potential as a once-a-week or once-amonth contraceptive, and as a drug to induce labor," says Schaff.

At present, 37 patients are receiving the drug 'off-label' for conditions other than abortion, under the Food and Drug Administration's "compassionate use" program.

\section{Marlene Cimons, Washington}

